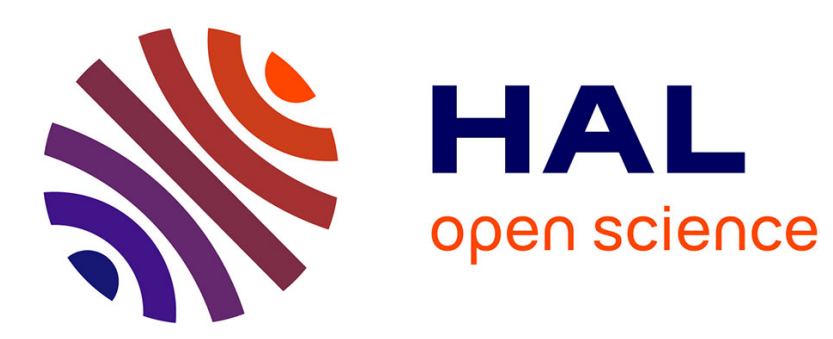

\title{
Source d'ions lourds multicharges triplemafios
}

\author{
P. Briand, N. Chan-Tung, R. Geller, B. Jacquot
}

\section{To cite this version:}

P. Briand, N. Chan-Tung, R. Geller, B. Jacquot. Source d'ions lourds multicharges triplemafios. Revue de Physique Appliquée, 1977, 12 (8), pp.1135-1138. 10.1051/rphysap:019770012080113500 . jpa-00244290

\section{HAL Id: jpa-00244290 https://hal.science/jpa-00244290}

Submitted on 1 Jan 1977

HAL is a multi-disciplinary open access archive for the deposit and dissemination of scientific research documents, whether they are published or not. The documents may come from teaching and research institutions in France or abroad, or from public or private research centers.
L'archive ouverte pluridisciplinaire HAL, est destinée au dépôt et à la diffusion de documents scientifiques de niveau recherche, publiés ou non, émanant des établissements d'enseignement et de recherche français ou étrangers, des laboratoires publics ou privés. 


\title{
SOURCE D'IONS LOURDS MULTICHARGES TRIPLEMAFIOS (*)
}

\author{
P. BRIAND, N. CHAN-TUNG (**), R. GELLER et B. JACQUOT \\ Association EURATOM-CEA \\ Département de Physique du Plasma et de la Fusion Contrôlée \\ Service IGn, Centre d'Etudes Nucléaires \\ 85 X, 38041 Grenoble Cedex, France
}

(Reçu le 3 mars 1977, accepté le 29 avril 1977)

\begin{abstract}
Résumé. - On décrit le principe de fonctionnement et les performances actuelles de la source. On indique les états de charges ioniques, les courants et les émittances globales du faisceau.

Abstract. - The principle and the characteristics of the ion source are described. We also furnish the up-to-date performances concerning the ion charge states, ion currents and global emittances of the beam.
\end{abstract}

1. Généralités sur les caractéristiques du plasma de la source Triplemafios. - Afin de prolonger le temps de fonctionnement sans interruption on a intérêt à s'affranchir de l'arc qui est la base de la plupart des sources d'ions. Dans notre source les électrons ne sont pas fournis par une cathode à durée de vie limitée mais par un plasma froid donc indestructible et ils ne sont pas accélérés par une tension d'arc mais par une onde électromagnétique résonnante de fréquence $\omega_{\mathrm{HF}}=\omega_{\mathrm{c}}^{-}\left(\omega_{\mathrm{c}}^{-}=e B / m^{-}\right.$est la fréquence de résonance cyclotronique des électrons de masse $m^{-}$ et charge $e$ dans un champ magnétique $B)[1,2,3]$. De ce fait on élimine les effets destructeurs de l'impact sur les électrodes ce qui rend le temps de vie de la source pratiquement illimité et cela même en régime continu. En réglant la puissance HF on peut obtenir une grande souplesse dans l'ajustement des paramètres primordiaux qui sont : la densité $n^{-}$et l'énergie $T^{-}$ des électrons du plasma, $N^{0}$ la densité du gaz neutre nécessaire au fonctionnement et $\tau^{+}$le temps de vie des ions en présence de ces électrons. Par ailleurs la source peut être pulsée à toutes les cadences en agissant soit sur la puissance HF, soit sur les tensions d'extraction.

On sait que pour obtenir des ions à charge $Z$ très élevée, il faut maximaliser le produit $n^{-} \tau^{+}$ $\left(>10^{9} \mathrm{~cm}^{-3} . \mathrm{s}\right)$ minimaliser le rapport $\left(N^{0} / n^{-}\right)$ $\left(<10^{-2}\right)$ et chauffer les électrons à $1<T^{-}<10 \mathrm{keV}$ afin qu'ils puissent ioniser dans les couches profondes des atomes, soit par collision unique soit par collisions multiples entre électrons et atomes déjà ionisés. (Le deuxième type de collisions étant plus efficace.) Il en résulte que les caractéristiques d'un tel plasma ne sont pas banales et mettent en jeu des densités d'énergie

(*) Communication présentée au Congrès National de Physique des Plasmas, Paris, 6-10 décembre 1976.

(**) GANIL. électronique considérables. Comme $\tau^{+}$dans les structures les plus élaborées ne dépasse guère $10^{-2} \mathrm{~s}$, on en conclut que $n^{-}$doit être $>10^{11} \mathrm{~cm}^{-3}$. Nos mesures interférométriques donnent en effet $\sim 3 \times 10^{11} \mathrm{~cm}^{-3}$ dans le $2^{\mathrm{e}}$ étage $\mathrm{HF}$ et $T^{-}$est réglable dans les limites indiquées. Il en résulte que la densité d'énergie électronique $n^{-} k T^{-}$se situe entre $3 \times 10^{14}$ et $3 \times 10^{15} \mathrm{eV} / \mathrm{cm}^{3}$, résultat confirmé par ailleurs par nos mesures de diamagnétisme. La source fonctionnant en régime continu, le flux de puissance électronique nécessaire à la formation d'un tel plasma est $\Phi_{\mathrm{p}}=\left(n^{-} k T^{-}\right) \cdot v^{-}$. Or la vitesse électronique $v^{-} \simeq 2 \times 10^{9} \mathrm{~cm} / \mathrm{s}$ pour $T^{-}>1 \mathrm{keV}$, il en résulte que $\Phi_{\mathrm{p}}$ vaut au minimum $6 \times 10^{23} \mathrm{eV} / \mathrm{cm}^{2}$.s soit $>100 \mathrm{~kW} / \mathrm{cm}^{2}$.

$\mathrm{Si}$ on veut alors extraire de ce plasma disons $5 \times 10^{13}$ ions/s dans l'état de charge le plus abondant $<Z>$ (qui représente environ $10 \%$ du total) il faudra extraire au total $\sim 5 \times 10^{14}$ ions/s; $s$ étant la surface $\mathrm{du}$ trou d'extraction, on devra satisfaire à la relation

$$
n^{+} v^{+} s / 4 \simeq 5 \times 10^{14} \simeq 1 / 4 \frac{n^{-}}{<Z>} v^{+} s .
$$

Sachant que la vitesse thermique des ions $v^{+}$est de l'ordre de $10^{5} \mathrm{~cm} / \mathrm{s}, s$ vaudra nécessairement $\sim 1 \mathrm{~cm}^{2}$. Il en résulte que la section du plasma de source doit dépasser $1 \mathrm{~cm}^{2}$ et que la puissance continue électrique $P$ qu'il faudra coupler aux électrons dépassera nettement $100 \mathrm{~kW}$. Ceci entraînerait de graves problèmes de dissipation thermique sur les parois. Heureusement qu'il existe une parade à ces difficultés : en effet il suffit de faire passer les mêmes électrons un grand nombre de fois par le même plan pour satisfaire $\Phi_{\mathrm{p}} \sim 100 \mathrm{~kW} / \mathrm{cm}^{2}$ tout en diminuant notablement $P$. C'est vers cette solution que nous nous sommes orientés quand nous confinons les électrons dans la structure magnétique à $B$ minimum du deuxième étage de la source. En permettant aux électrons énergétiques 
d'effectuer une centaine d'aller et retour dans cette structure, il est devenu possible d'assurer l'entretien du plasma considéré à l'aide d'une puissance $P=P_{\mathrm{HF}_{2}}$ de l'ordre du kilowatt. La configuration magnétique qui fournit ces performances est composée d'une bouteille magnétique classique à laquelle on superpose un champ magnétique hexapolaire. Afin de satisfaire en dynamique aux conditions de pression $(p \lesssim 2 \times$ $10^{-7}$ torr) dans la structure, cette dernière doit permettre des pompages efficaces. Il en résulte que la

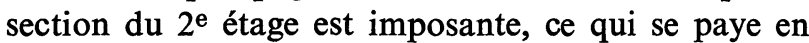
consommation électrique ( $3 \mathrm{MW}$ ) pour établir le volume magnétique nécessaire (une solution supraconductrice pourrait réduire cette consommation de $80 \%$ à $90 \%$ ).

2. Description de la source Triplemafios. - Notre source se caractérise donc par ses grandes dimensions géométriques, offrant des conductances adéquates pour les pompages le long du dispositif. (Au total plus de $10000 \mathrm{l} / \mathrm{s}$ par pompe à diffusion et $30000 \mathrm{1} / \mathrm{s}$ par pompe à titane.) Le dispositif opère en 3 étages (Fig. 1). Un $1^{\mathrm{er}}$ étage, injecteur de plasma (pression

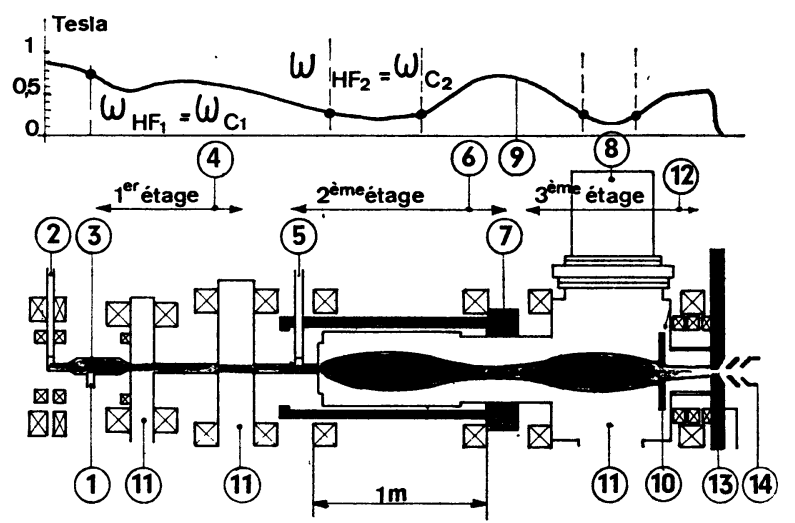

Fig. 1. - TRIPLEMAFIOS : 1, injection de gaz; 2, arrivée $\mathrm{HF}_{1}(16 \mathrm{GHz}) ; 3$, source du plasma d'injection ; 4, $1^{\text {er }}$ étage $\left(p \sim 10^{-3}\right.$ torr $) ; 5$, arrivée $\mathrm{HF}_{2}(8 \mathrm{GHz}) ; 6,2^{\circ}$ étage $\left(p \sim 10^{-7}\right.$ torr) ; 7 , bobine hexapolaire $; 8$, pompage à titane $; 9$, champ magnétique axial avec plans de résonance R. C. E. ; 10, diaphragme ; 11 , pompages $; 12,3^{e}$ étage $; 13$, blindage ferromagnétique avec cuvette d'expansion; 14, extracteur ionique. [TRIPLEMAFIOS : 1 , gas injection ; $2, \mathrm{RF}_{1}$ terminal $(16 \mathrm{GHz})$; 3 , plasma injector ; $4,1^{\text {st }}$ stage $\left(p \sim 10^{-3}\right.$ torr $) ; 5, \mathrm{RF}_{2}$ terminal $(8 \mathrm{GHz}) ; 6,2^{\text {nd }}$ stage $\left(p \sim 10^{-7}\right.$ torr $) ; 7$, hexapolar coil ; 8, titanum pump ; 9, axial magnetic field with E. C.R. planes ; 10 , diaphragm $; 11$, vacuum pumping $; 12,3^{\text {rd }}$ stage $; 13$, ferromagnetic shield with expansion cup ; 14, ion extractor.]

gaz $10^{-2}-10^{-4}$ torr) où l'on crée par une première résonance gyromagnétique (puissance $\mathrm{HF}_{\mathbf{1}}$ utile $<1 \mathrm{~kW}, \omega_{\mathrm{HF}_{1}}=\omega_{\mathrm{CE}_{1}}=2 \pi \times 16 \times 10^{9} \mathrm{~Hz}, B_{1} \sim 0,6$ tesla) un plasma froid mais pouvant déjà contenir des ions faiblement strippés. Il n'y a donc, ni filaments, ni électrodes dans cet injecteur.

Après un pompage différentiel (pression de gaz $10^{-7}$ torr) un $2^{\mathrm{e}}$ étage est constitué par la structure magnétique à $\mathbf{B}$ minimum vers laquelle diffuse le plasma d'injection et dont les électrons subissent une $2^{e}$ résonance gyromagnétique $\left(P_{\mathrm{HF}_{2}}\right.$ utile de 1 à $5 \mathrm{~kW}, \omega_{\mathrm{HF}_{2}}=\omega_{\mathrm{CE}_{2}}=2 \pi \times 8 \times 10^{9} \mathrm{~Hz}, B_{2} \sim 0,3$ tesla). Cette résonance chauffe les électrons ( 1 à $10 \mathrm{keV})$, par force ambipolaire les ions et électrons sont retenus un certain temps dans la structure magnétique et les collisions ionisantes électrons/ions y font augmenter notablement le $\langle Z\rangle$ moyen ionique. Une partie de ce plasma diffuse alors vers un $3^{e}$ étage qui permet l'adaptation d'une extraction classique par cuvette d'expansion en champ magnétique faible. Cet étage permet en effet, en vue d'accroître le nombre d'ions à extraire, de reconcentrer la densité du plasma près de l'extracteur dans le col de la $3^{\mathrm{e}}$ bouteille magnétique. Pour éviter des aberrations incorrigibles de l'optique du faisceau ionique (théorème de Busch), nous diminuons brutalement par un blindage ferro-magnétique le champ magnétique dans l'espace d'extraction. C'est dans la masse même de ce blindage épais que se situe le cylindre d'expansion du plasma. Une telle géométrie n'est pas idéale pour la conservation des ions car elle favorise les recombinaisons sur la paroi et peut détériorer les abondances en ions fortement chargés. Par ailleurs elle rediminue par l'expansion volumique du plasma sa densité $n$ sur le ménisque d'extraction.

Mais elle représente un compromis avantageux car :

a) elle permet la réalisation d'une bonne émittance du faisceau extrait,

b) elle sépare mécaniquement le plasma où les ions se forment du faisceau où ils sont accélérés par champs électriques,

c) elle permet de doubler la longueur $L$ du plasma utile (c'est-à-dire contenant des électrons énergétiques) et par ce fait d'accroître le temps de diffusion des ions à travers le nuage d'électrons qui les épluche.

3. Performances de la source Triplemafios. - 3.1 EMITTANCE GLOBALE D'UN FAISCEAU D'IONS MULTICHARGÉS D'ARGON. - A l'aide d'un système de 4 électrodes

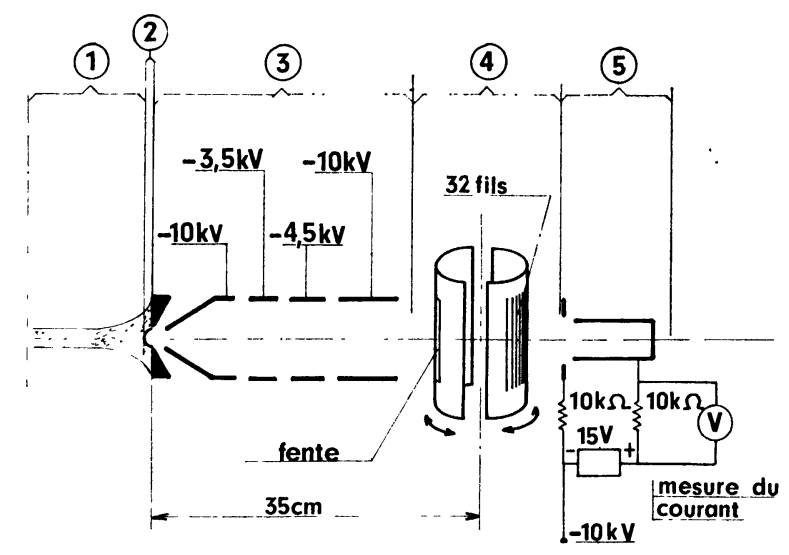

Fig. 2. - Schéma du montage de mesure de l'émittance globale du faisceau d'argon : 1 , cuvette d'expansion du plasma de source ; 2 , trou d'extraction et ménisque de plasma ; 3 , lentilles d'extraction et de transport; 4, émittancemètre à cylindres mobiles ; 5, cage de Faraday et anneau de garde.

[Scheme of the global argon beam emittance measurement : 1 , expansion cup of the source plasma ; 2 , extraction hole and plasma meniscus; 3 , extraction and transport electrodes; 4, mobile target emittance meter ; 5, Faraday cup and shielding ring.] 
représenté sur la figure 2, on extrait et transporte le faisceau. Les tensions appliquées aux électrodes sont : $\mathrm{E}_{1}$ et $\mathrm{E}_{4}:-10 \mathrm{kV}, \mathrm{E}_{2}:-3,5 \mathrm{kV}, \mathrm{E}_{3}:-4,5 \mathrm{kV}$. Le plasma ayant été réglé pour un fonctionnement de routine, on a utilisé 3 trous d'extraction de diamètre différent : $\varnothing=7,14$ et $20 \mathrm{~mm}$. Les courants totaux $I^{+}$ extraits en régime continu étaient respectivement de l'ordre de $\sim 12,65$ et $120 \mu \mathrm{A}$ (Fig. 3). Le chromatisme ionique qui a été mesuré auparavant est de l'ordre de $80 \mathrm{eV} \times Z$ [2]. La stabilité du courant pour le fonctionnement de routine peut être assurée sur des journées et la fidélité de reproduction des caractéristiques est très satisfaisante.

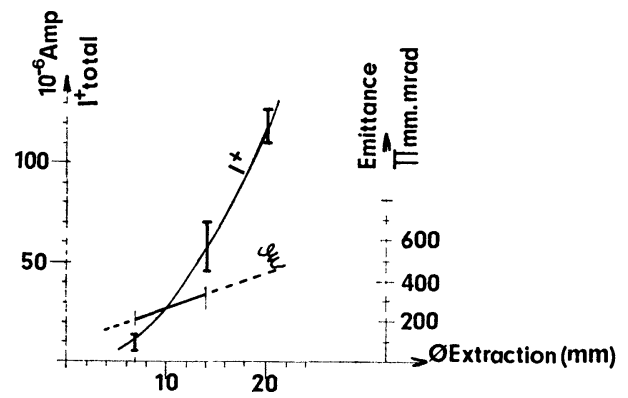

Fig. 3. - Courant ionique total et émittances du faisceau total en fonction de la dimension du trou d'extraction. [Total ion current and beam emittance versus extraction hole size.]

L'émittance $\varepsilon$ du courant global contenant tout le speetre de charge a été mesurée à $35 \mathrm{~cm}$ des trous d'extraction ayant 7 et $14 \mathrm{~mm}$ de diamètre. A plus de $90 \%$ de l'intensité on trouve respectivement 220 et $380 \pi . \mathrm{mm} . \mathrm{mrad}$ (Fig. 3). L'émittance d'un faisceau
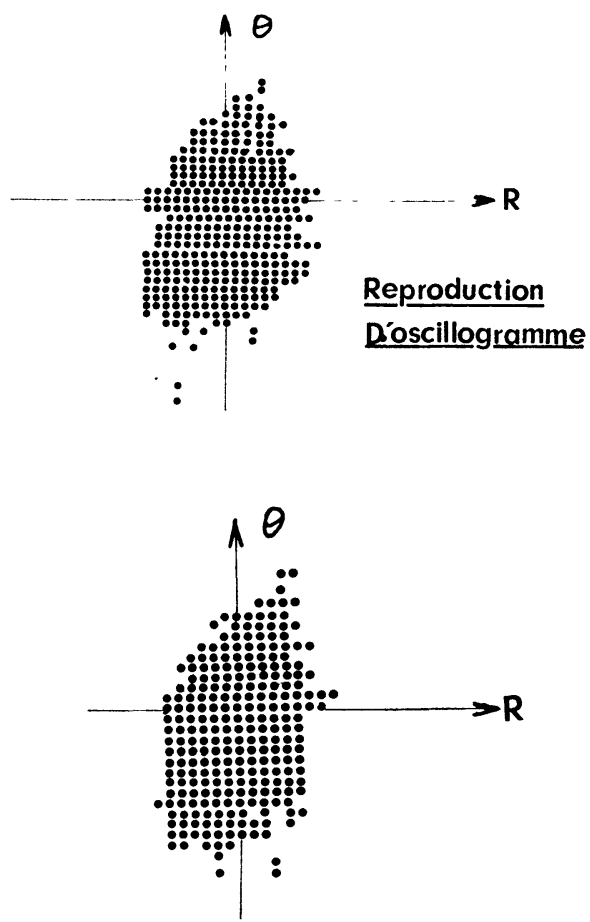

Figs. 4 et 5 . - Emittances globales à $90 \%$ et $80 \%$ du faisceau. [90\% and $80 \%$ global beam emittances.] sélectionné par état de charge ne pourra donc qu'être meilleure. Les figures 4 et 5 représentent les diagrammes d'émittance pour un courant de $50 \mu \mathrm{A}$ quand on considère 90 et $80 \%$ du courant total.

De nombreux diagrammes semblables ont été enregistrés et prouvent la fiabilité et la stabilité du faisceau en fonction du temps.

Si le champ magnétique $B$ dans l'espace du ménisque passe de $0,02 \mathrm{~T}$ à $0,1 \mathrm{~T}$ on voit l'émittance tourner mais la surface se conserve. Cette quasi-indépendance des qualités optiques de notre faisceau d'ions multichargés vis-à-vis de $B$ prouve que les forces exercées sur les ions par les grad $B$ présents dans l'espace d'extraction sont petites par rapport aux forces électriques.

$\varepsilon$ est mesuré par l'émittancemètre de Belmont de Bois décrit par ailleurs [4].

3.2 COURANT D'IONS MULTICHARGÉS ET SPECTRES D'ABONDANCE. - Pour mesurer les courants d'ions multichargés, l'émittancemètre est remplacé par un sélecteur magnétique rudimentaire (Fig. 6) ayant une

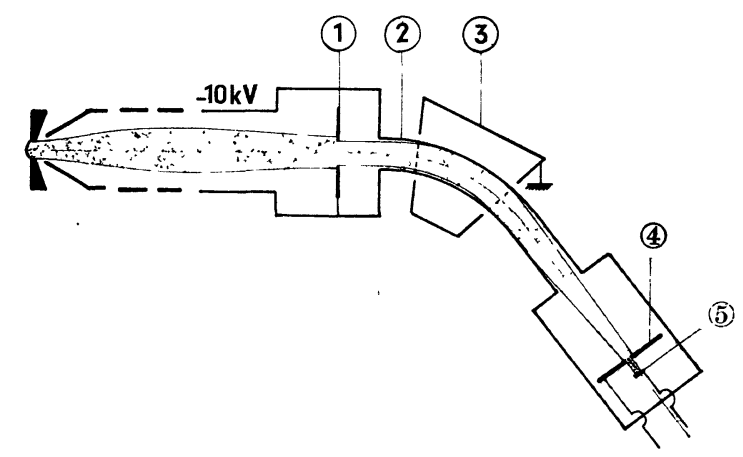

Fig. 6. - Analyse du faisceau : 1, fente d'entrée mobile ; 2 , tube analyseur à $-10 \mathrm{kV} ; 3$, électro-aimant ; 4 , fente de sortie ; 5, cage de Faraday.

[Beam analyser : 1 , mobile entrance slit ; 2 , analyser tube at $10 \mathrm{kV} ; 3$, electromagnet ; 4, output slit ; 5, Faraday cup.]

fente d'entrée de $2 \times 2,5 \mathrm{~cm}$ et une fente de sortie $1 \times 3 \mathrm{~cm}$ suivi d'une cage de Faraday. La figure 7 montre qu'avec une extraction de $10 \mathrm{kV}$, en régime continu, $\varepsilon$ étant $<400 \pi . \mathrm{mm}$. mrad, on obtient des

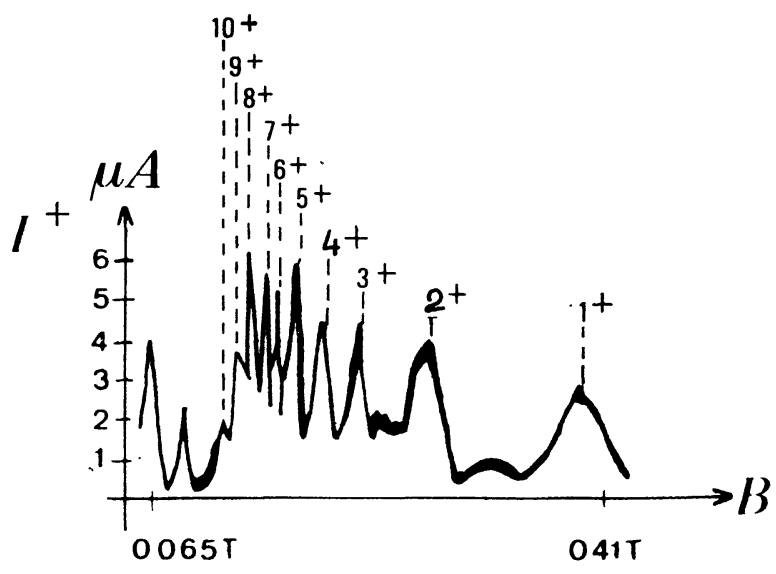

Fig. 7. - Spectre d'abondance des ions d'argon. [Argon ion abundance spectrum.] 
microampères d'ions $\mathrm{A}^{7+}, \mathrm{A}^{8+}, \mathrm{A}^{9+}$ et $\mathrm{A}^{10+}$ dans la cage. Environ $50 \%$ des particules se perdent dans le sélecteur où $P=2 \times 10^{-6}$ torr. En optimisant l'opti-

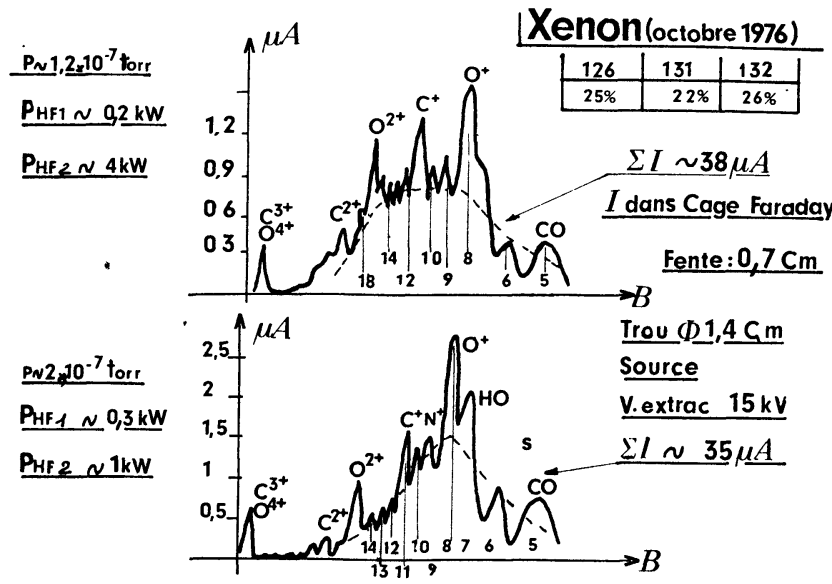

Fig. 8. - Spectre d'abondance des ions de xénon pour deux réglages différents.

[Xenon ion abundance spectrum for two different matchings].

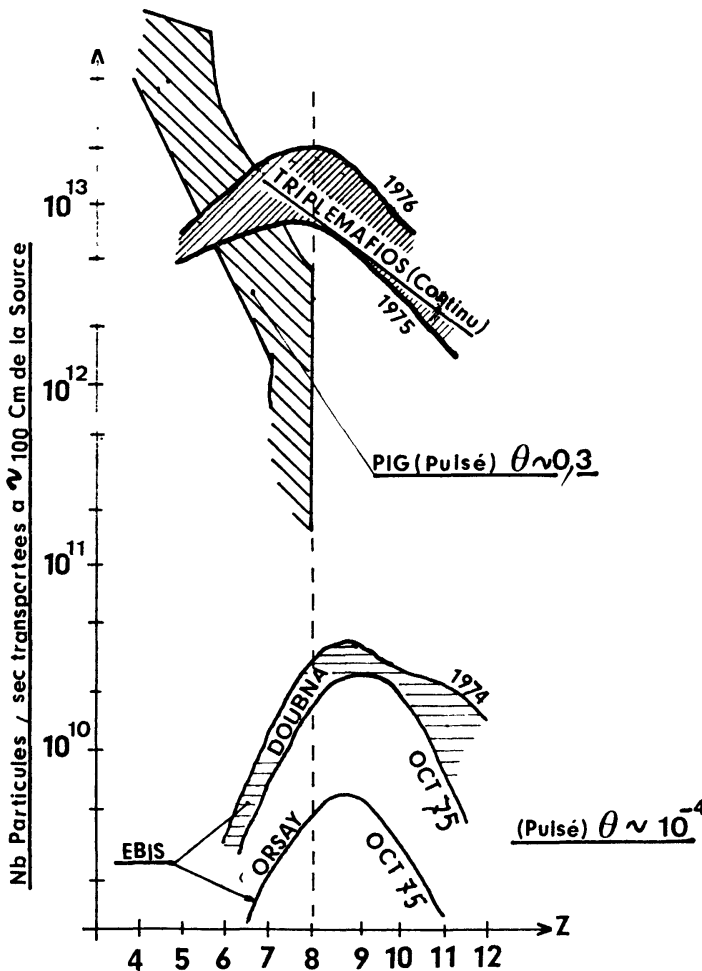

Fig. 9. - Comparaison entre performances de différentes sources d'ions multichargées pour l'argon.

[Comparison between the performances of different multiply charges ion sources for argon.] que du faisceau, dans l'état actuel on a pu mesurer $\sim 20 \mathrm{~mA}$ d'ions $\mathrm{A}^{8+}$ avec $15 \mathrm{kV}$ d'extraction. Un ensemble à double séparation magnétique est en voie de réalisation et permettra d'accroître ces performances.

La figure 8 montre des spectres de xénon obtenus par ce même dispositif.

La figure 9 donne les performances en ions d'argon pour différentes sources d'ions multichargés $[5,6,7]$.

La figure 10 résume les performances de Triplemafios par rapport aux sources P. I. G. On voit que l'état de charge le plus abondant est environ deux fois celui des P. I. G. Comme $\langle Z\rangle$ varie avec $\log \left(n^{-} \tau^{+}\right)$on peut en déduire que le facteur $n^{-} \tau^{+}$ dans le plasma de Triplemafios est environ 10 fois supérieur à celui des P. I. G. [8].

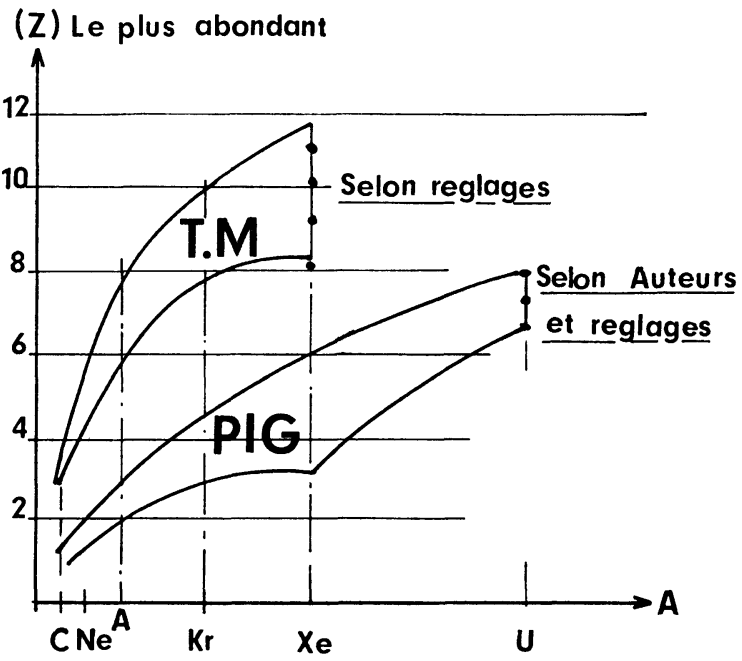

Fig. 10. - Comparaison entre TRIPLEMAFIOS et P.I.G. pour différents éléments.

[ $Z$ average comparison between Triplemafios and P. I. G. for different elements.]

\section{Valeurs ty.pes du Nb de particules/s ex traites et transportées}

\begin{tabular}{|c|c|c|c|c|c|c|c|c|c|}
\hline$C_{3^{*}}$ & $C_{5^{*}}$ & $O_{4^{*}}$ & $O_{7^{*}}$ & $\mathrm{Ne}^{*}$ & $\mathrm{Ne}_{8^{*}}$ & $A_{8^{*}}$ & $A_{10^{*}}$ & $X_{e_{10}}$ & $X_{e_{18^{*}}}$ \\
\hline $10^{14}$ & $2 \times 10^{13}$ & $10^{14}$ & $10^{13}$ & $5_{k} 10^{13}$ & $5_{x} 10^{12}$ & $2_{4} 10^{13}$ & $6_{x} 10^{12}$ & $10^{12}$ & $3_{x} 10^{11}$ \\
\hline
\end{tabular}

FIG. 11.

Enfin le tableau figure 11 indique quelques valeurs typiques pour le nombre de particules extraites de la source Triplemafios et transportées à environ $1 \mathrm{~m}$ dans l'état actuel de l'expérience.

\section{Bibliographie}

[1] Briand, P., Geller, R., Jacquot, B., Jacquot, C., Theiss, A., C. R. Hebd. Séan. Acad. Sci. 280 B (1975) 711.

[2] Geller, R., Nucl. Sci. 23 (1976) 904.

[3] Briand, P., Geller, R., JaCQUot, B., JacQuot, C., Nucl. Instrum. Methods 131 (1975) 407.

[4] Belmont, J. L., BoIs, J. M., Emittancemètre rapide, I. S. N. RI 7641 (1976).
[5] Bex, L., Clark, P. J., Ellisworth, C. E., Estrella, R. M., Gough, R., Holley, W. R., Nucl. Sci. 23 (1976) 107.

[6] Donets, E. D., Nucl. Sci. 23 (1976) 897.

[7] Arianer, J., Goldstein, C., Nucl. Sci. 23 (1976) 979.

[8] Daugherty, D., Eninger, J. E., Janes, G. S., Levy, R. H., Res. Rep. 333 Avco Everett Res. Lab. (1969). 